\title{
Developing a Mathematical Model for Hydrate Formation in a Spray Batch Reactor
}

\author{
Mohammad Kazemeini ${ }^{1}$, Farideh Freidoonian ${ }^{2}$, Moslem Fattahi ${ }^{1}$ \\ ${ }^{1}$ Department of Chemical and Petroleum Engineering, Sharif University of Technology, Tehran, Iran \\ ${ }^{2}$ Islamic Azad University, South Tehran Branch, Tehran, Iran \\ Email: kazemini@sharif.edu, freidoonianf@yahoo.com,moslem.fattahi@gmail.com
}

Received 2012

\begin{abstract}
The formation of methane hydrate was undertaken in this research. The purpose of this work was to model the methane hydrate formation with a hydrate-water-methane system in a semi-batch reactor under steady-state, isothermal and isobaric conditions. Obtained results were validated with experiments conducted in a semi-batch spray reactor at low temperatures and high pressures. The investigated formation of gas hydrate from pure methane required physical constants of these materials which were determined through experimental data. The experiments hence, the theoretical calculations were conducted with pure methane and carried out in a spray reactor at $273.95 \mathrm{~K}$ and $8705 \mathrm{kPa}$ to determine the actual amount of hydrate formation in such reactor. Ultimately; the comparison of the results generated from the developed mathematical model with those of experimental data of others indicated a very satisfactory agreement obtained.
\end{abstract}

Keywords: Hydrate Formation; Methane; Spray Reactor; Semi-batch; Modeling

\section{Introduction}

Hydrate are crystalline water-based solids physically resembling ice, in which low molecular weight (light) gas molecules are trapped by water molecules bounded by hydrogen and stabilized due to Van-der-waals forces. In hydrate formation host molecule is water and guest molecule is a gas or liquid [1].

Thermodynamic conditions of hydrate formation are often found in pipelines. It is unfavorable because these crystals might plug the flow line and damage valves and instrumentation. Hydrate formation within pipelines slows down the flow of materials due to blockage causing significant economic losses. Hydrate might be used for gas storage and transportation as well. Utilizing hydrate as a storage mean for transportation depends upon the maximum gas storable through hydrate and the hydrate formation rate. Research in this area started at beginning of the 1990s. Gudmundsson and his group at Norwegian University reported results of experimental investigations on production, storage and transportation of gas hydrates [2,3].

The hydrates are to crystallize in 3 structures, I, II and $\mathrm{H}$, depending upon the nature and the size of guest molecules [4, 5]. A unit cell of structure I contained eight cavities (two smalls and six large ones) and formed by 46 hydrogen-bonded water molecules. While a unit cell of structure II contained 136 water molecules and enclosed 24 cavities including 16 smalls and 8 large ones. The gas molecules of methane, ethane, propane, isobutene, $\mathrm{CO}_{2}, \mathrm{H}_{2} \mathrm{~S}$, and $\mathrm{N}_{2}$ were known to stabilize the micro cavities formed by either of the two hydrate structures.

The formation of either of structures I or II is related to the ratio of the guest molecule to the cavity size while the thermodynamic operating conditions including temperature, pressure and gas composition are also very important affecting formation of these structures.
The kinetic models of hydrate formation studied previously have been developed based upon stirred tank batch systems [6]. Such reactors included water at hydrate formation conditions being injected along with the gas for production of the hydrates. Natural gas hydrate formation condition was usually determined experimentally in the laboratory however; such data were not always available. Hence, correlations utilized in order to determine values for the natural gas hydrate formation conditions.

Hydrate formation kinetics usually improved through addition of a promoter. One of the most popular promoters was paratoluenesulfonic acid (pTSA) which was undertaken in the present research. The work reported here therefore; described a theoretical analysis for the methane hydrate formation in presence of the pTSA, the results for which were compared with experimental data available in the open literature to verify the model.

\section{Theoretical Background}

The mathematical model of the hydrate formation in this work included two main steps of: (1) nucleation and growth modeling of hydrate and (2) modeling of a semi-batch spray reactor process. The presence of non-polar molecules such as hydrocarbons in water distorted water molecules to organize themselves into clusters forming the needed nuclei. The nucleation time named induction period. Furthermore, due to the highest concentration of gas at the water-gas interface, the hydrate formation took place at this location. The process to be modeled was then a semi-batch heterogeneous spray reactor for methane hydrate production into a pressurized vessel. This process involved a spraying period during which the reactor operated in a semi-batch mode and a stabilization period during which the 
reactor operated in a batch mode.

\subsection{Materials}

The main component of methane hydrate was water which occupied around $80 \%$ by mass and the remainder devoted to methane. The simulated promoter mixed with water was the pTSA with predetermined concentration of $1.25 \mathrm{~g} / \mathrm{l}$. Pure methane was also utilized to form hydrates.

\subsection{Apparatus}

Figure 1 showed a schematic drawing of the experimental apparatus leading to the present investigation. A known amount of water was fed into the reactor, which was purged continuously with the pure methane. The reactor operated in a semibatch mode. A fixed stabilization period of $5 \mathrm{~min}$ was maintained after spraying, where the reactor behaved as a batch system to form the product [7].

\section{Computational Procedures}

In this simulation the MATLAB software of version 2011 to calculate the correlations of hydrate formation was utilized. As mentioned above, initial phase of hydrate formation was semi-batch water injection or spraying process, and the second phase was a batch process requiring stabilization period. The injection time, $\mathrm{t}_{\mathrm{i}}$, for spraying period was determined to be 798s and the induction time, $t_{s}$, for stabilization period was found to be 370s. Water molecules with initial velocity were accounted for to travel the horizontal distance thru the reactor. During this traversal, the methane was injected inside the reactor to form the hydrate. The reaction between water molecules and methane was a physical interaction. The hydrate formation reaction equation might have been written as:

$$
\text { GAS + (1/B) WATER = HYDRATE }
$$

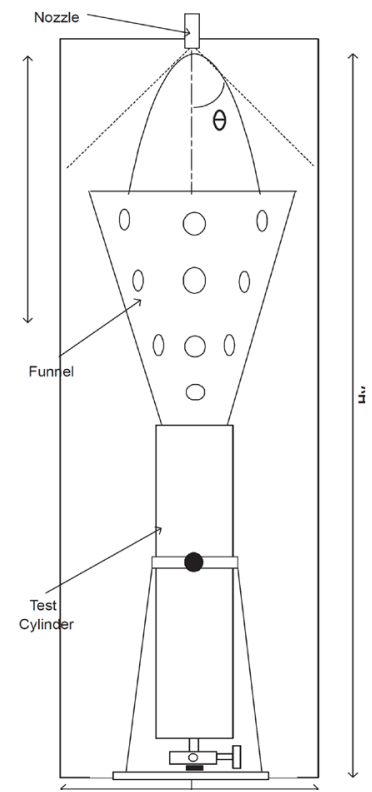

Figure 1. Schematic diagram of the spray reactor leading to hydrate formation.
In which $\mathrm{B}$ was the equilibrium gas to water molar ratio. The constant for calculating the equations were based upon the experimental data. As mentioned, in this model operating condition were taken to be isothermal, hence only mass transfer during hydrate formation existed and respective calculations were performed accordingly. The model parameters were of geometrical dimensions and physical constants used in developing the model [7].

In the present work, through solving equations involving hydrate formation, the theoretical data were calculated then compared with experimental ones.

\subsection{Governing Equations}

The distance travelled $\mathrm{x}(\mathrm{t})$ by water in reactor was calculated from following correlation [7]:

$$
\frac{d^{2} x}{d t^{2}}=4.41-0.093\left(\frac{d x}{d t}\right)^{2}
$$

The boundry conditions were:

$$
\mathrm{t}=0 \text {, at } \mathrm{x}=0 \text { and } \mathrm{v}=\mathrm{v}_{0}
$$

The constant of this correlation was calculated by MATLAB software with model parameters related to hydrate formation kinetics.

By water molecules travelling the horizontal distance thru the reactor (x), the initial velocity decreased. Hence, the acceleration subsequently lowered as well with the following equation:

$$
a V=\frac{h t}{t_{i}}
$$

In which ht was the distance travelled by the spray flow.

The diffusion coefficient of gas in water for hydrate formation was calculated through the following equation:

$$
D_{c}=\frac{7.4 \times 10^{-8}\left(M W_{w}\right)^{0.5} \times T}{\mu_{w} V_{m}^{0.6}}
$$

where $\mu_{\mathrm{w}}$ is the associate factor of solvent (i.e.; water) and $\mathrm{V}$ is the molar volume.

Mass transfer coefficient of methane in water during spraying was provided by:

$$
K_{L}=\frac{0.16 D_{c}}{d_{p}} S c^{0.5} W e^{0.5} \mathrm{Re}^{5 / 16}
$$

Supersaturation, $\Delta \mu$, was the driving force for hydrate formation where it was the difference between the chemical potentials of the old and new phases. For hydrate nucleation this parameter should be $\Delta \mu=0$ and for a single gas component system such as methane it was calculated as follows:

$$
\Delta \mu=K T \ln \frac{\phi\left(P_{r}, T\right) P_{r}}{\phi\left(P_{e}, T\right) P_{e}}+\Delta v_{e}\left(P_{r}-P_{e}\right)
$$

When water mixed with the hydrate, a promoting chemical was sprayed into the reactor and maintained at subzero temperatures at which hydrate as well as; ice nucleations occurred together. The nucleation rate was determined as follows:

$$
J=A \exp \exp \left(\frac{\Delta \mu}{K T}\right) \exp \left(\frac{-4 c^{3} v_{h e f}^{23}}{27 k T \Delta \mu^{2}}\right)
$$


where $A$ is the kinetic parameter for nucleation. $V_{h}$ is velocity of hydrate and $d_{e f}$ is the effective surface energy.

The concentration of water, $\mathrm{C}_{\mathrm{w}}(\mathrm{t})$, at any given time during water injection could be determined from the following:

$$
t \frac{d C_{w}(t)}{d t}+\left(1+K e_{1}(t) t\right) C_{w}(t)=C_{w}^{0}
$$

In which, $\mathrm{K}_{\mathrm{e}}$ is the varying rate constant used to determine $\mathrm{C}_{\mathrm{w}}$. The moles of water crystallized, during semi-batch and batch process might be calculated as:

For semi-batch period at $\mathrm{t} \leq \mathrm{t}_{\mathrm{i}}$ :

$$
M_{w}^{c}(t)=\left(C_{w-}^{0} c_{w}(t)\right) Q t
$$

For batch period at $\mathrm{t}=\mathrm{t}_{\mathrm{i}}$ :

$$
M_{w}^{c}(t)=M_{w}^{c}(t i)\left[1+\left(K e_{2}(t-t i)\right)(t-t i)\right]
$$

According to the above equations one might observe the growth of water crystallized with time. Now, the moles of water crystallized and remained as well as; that of the gas farmed into hydrate in vessel might be determined.

The relationship for moles of gas in hydrates at any time is given by:

$$
M_{g}^{h}(t)=M_{w}^{c}(t) B
$$

Water remaining in the system would be in the form of ice the formation of which was inevitable in the spray reactor operation due to the low temperature, where could be written as:

$$
M_{w}^{r}(t)=M_{w}^{i}(t)-M_{w}^{c}(t)
$$

where, $M_{w}^{i}(t)$, is the initial moles of water in the system. So the moles of gas remaining in the vessel are given as:

$$
M_{g}^{r}(t)=M_{g}^{0}(t)-M_{g}^{h}(t)
$$

where, $M_{g}^{0}(t)$, is the total moles of methane in the system at $\mathrm{t}=\mathrm{t}_{0}$.

The gas to total water volume ratio, HFVF, or hydrate volume factor assuming the ideal gas law could be calculated as:

$$
\operatorname{HFVF}(t)=\frac{M_{g}^{h}(t)\left(R c\left(\frac{T_{s}}{P_{s}}\right)\right.}{Q t_{i}}
$$

Details of all correlation and equation for hydrate formation kinetics were presented in Ref. [7].

\section{Results and Discussion}

According to the Equation (1), the distance travelled by water in vessel, X, was shown in Figure 2.

According to correlation (2), the hydrate acceleration, a, in reactor with time decreased. The variations of acceleration with time were showed in Figure 3.

The initial water in the system, $\mathbf{M}_{\mathrm{w}, \mathrm{i}}$, at any given time was presented in Figure 4.

The water crystallized into hydrate form, $\mathrm{M}_{\mathrm{c}, \mathrm{w}}$, was calculated through the correlation (9) and shown in Figure 5. The water remained in vessel was ice.

The mole fraction of methane in hydrate was about 0.2. The equation (10), mole of methane in hydrate, $\mathbf{M}_{\mathrm{gh}}$, was displayed in Figure 6:
The amount of gas in hydrate was indeed less than water hence, the variation of remaining gas with time, $\mathrm{M}_{\mathrm{gr}}$, was also low. This was calculated through the equation (11) and shown in Figure 7.

Hydrate formation volume factor, HFVF, or the gas total water volume ratio was determined from correlation (13) and shown in Figure 8.

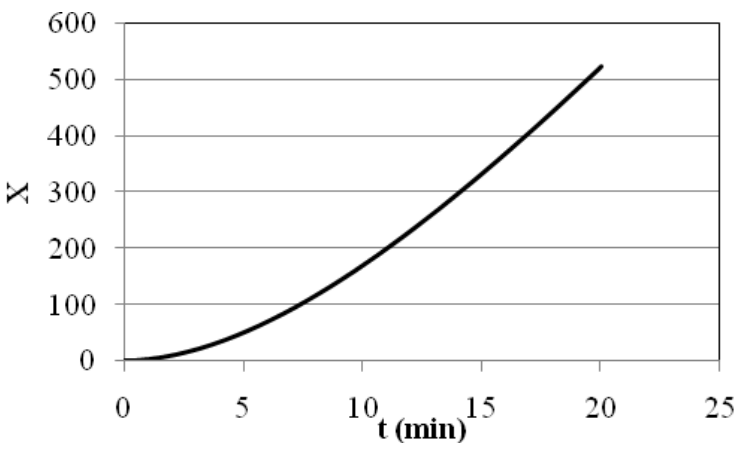

Figure 2. Variation of material's travelled distance with time.

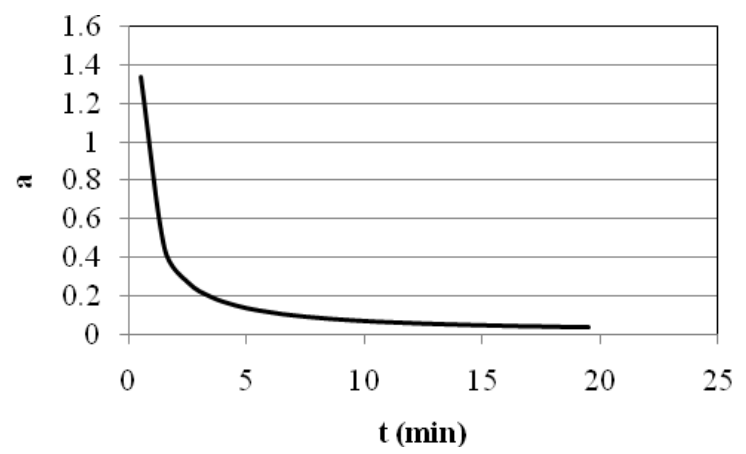

Figure 3. Material's acceleration variation with time.

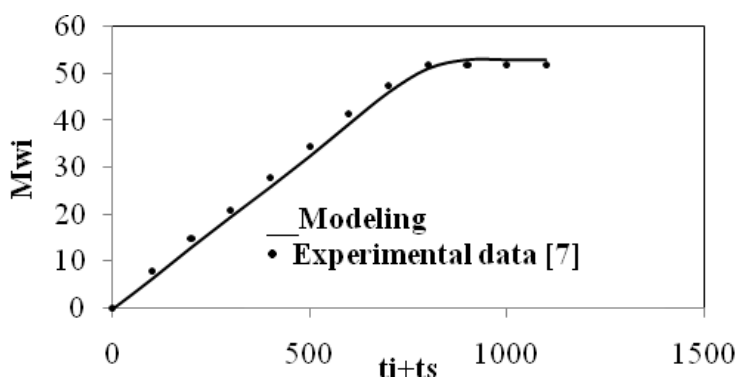

Figure 4 . The initial mole of water injected variation with time.

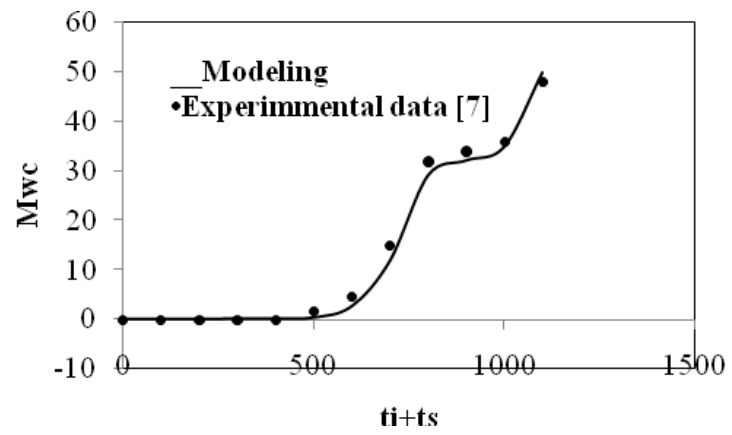

Figure 5. The mole of water crystallized variation with time. 


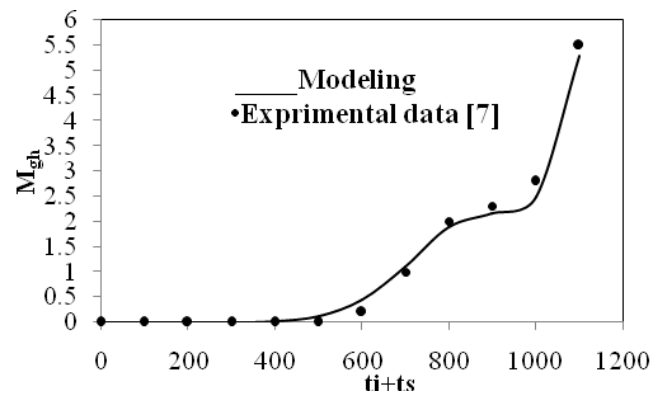

Figure 6. Variation of the mole of methane formed in the hydrate with time.

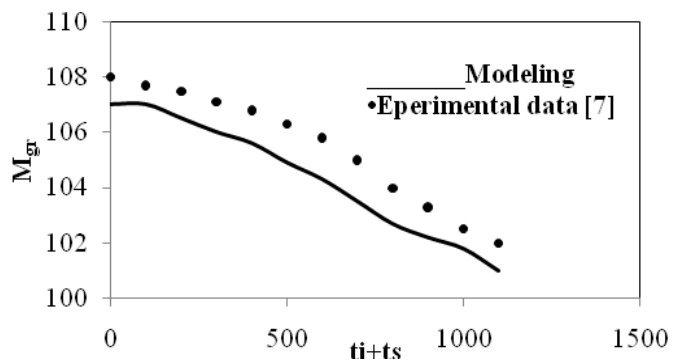

Figure 7. Variation of the mole of remaining gas in the vessel with time.

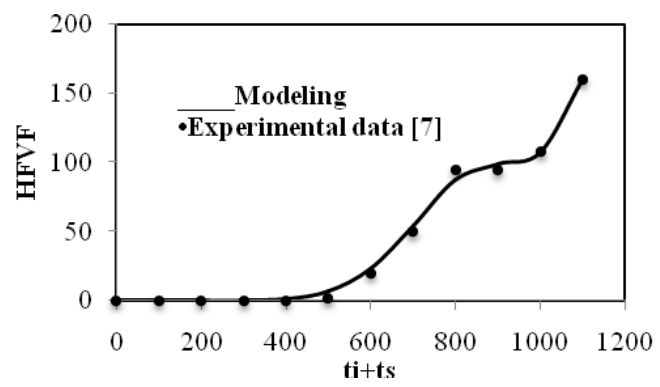

Figure 8. The HFVF variation with time.

The error percentage in terms of absolute error between experimental and theoretical (or calculated) data generated from the developed model was reported in Table 1 in order to consider the accuracy of the hydrate formation model put together in this research.

It is seen through the above figures that at $t=798$, due to changes in the governing equations of hydrate formation leading to variations in spraying and stabilization periods, one might see fractures in the behaviors displayed. Nonetheless; the

Table 1. The AAD and AE of effecting parameters.

\begin{tabular}{cccc}
\hline \multirow{2}{*}{ Quantity } & \multicolumn{3}{c}{ Percentage of Error } \\
\cline { 2 - 4 } & $\begin{array}{c}\text { Absolute Error } \\
\text { (\%) }\end{array}$ & $\begin{array}{c}\text { AAD } \\
\text { (Experimental) }\end{array}$ & $\begin{array}{c}\text { AAD } \\
\text { (Modeling) }\end{array}$ \\
\hline $\mathrm{M}_{\mathrm{wi}}$ & 6.45 & 5.70 & 5.37 \\
$\mathrm{M}_{\mathrm{wc}}$ & 10.42 & 12.56 & 13.37 \\
$\mathrm{M}_{\mathrm{gr}}$ & 0.90 & 0.19 & 0.20 \\
$\mathrm{M}_{\mathrm{gh}}$ & 10.27 & 12.91 & 11.59 \\
$\mathrm{HFVF}$ & 6.42 & 16.40 & 12.62 \\
\hline
\end{tabular}

AAD (Average Absolute Deviation) and AE (Absolute Error) values between theoretical and experimental extents of several key affecting parameters displayed in Table $\mathbf{1}$ revealed that the accuracy of the developed model might be considered rather satisfactory.

\section{Conclusions}

The following behavioral patterns were demonstrated by this investigation:

- When hydrate molecules traveled thru horizontal distance of the reactor, their average flight velocity increased while their acceleration decreased.

- The increase in the water injected showed an enhancement in the hydrate formation because as mentioned, the highest volume of hydrate was related to the water species.

- Increase in the stabilization period enhanced hydrate amount formation.

- During induction time period, hydrate formation occurred and an exponential hydrate crystal growth followed.

- The initial amount of water in the reactor increased with time since the most important component of the hydrate was indeed the water. In other words, not only the mole fraction of this species in the hydrate was $80 \%$ but also, it was injected into the reactor with passage of time.

- As time went by, the moles of methane in system decreased rather slowly due to the low initial consumption (i.e.; about $5 \%$ of the total initial moles) of this species

All of these issues were indicative of how the chemistry and physics of the hydrate material affected its displayed behavior. This model paved down the road toward further optimization of hydrate formation process and its applications which are currently undertaken in this laboratory.

\section{REFERENCES}

[1] D. Kashchive, and A. Firoozabadi, "Driving force for crystallization of gas hydrates,” Journal of Crystal Grwoth, vol. 241, pp. 220-230, 2002.

[2] J.S. Gudmundsson, V. Andersson and O.I. Levik, "Gas storage and transportation using hydrates," Offshore Mediterranean Conference, Ravenna, March 19-21, 1997.

[3] J.S. Gudmundsson, and A. Børrehaug, "Frozen hydrate for transport of natural gas," 2nd International Conference on Natural Gas Hydrate, June 2-6, Toulouse, France, 1996.

[4] E.D. Solan, and C.A. Koh, "Clathrate hydrate of natural gases," 2nd Edition, New York; Taylor \& Francis, 2007.

[5] M. A. Clarke, and P.R. Bishno, "Measuring and modelling the rate of decomposition of gas hydrates formed from mixtures of methane and ethane," Chemical Engineering Science, vol. 56, pp. 4715-4724, 2001.

[6] P. Englezos, N. Kalogerakis, P.D. Dholabhai, and P.R. Bishnoi, "Kinetics of formation of methane and ethane gas hydrates," Chemical Engineering Science, vol. 42, pp. 2647-2658, 1987.

[7] N. Gnanendran, and R. Amin, "Modelling hydrate formation kinetics of a hydrate promoter-water-natural gas system in a semi-batch" Chemical Engineering Science, vol. 59, pp. 3849-3863, 2004. 